\title{
On R\&D Information Sharing and Merger
}

\author{
Uday Bhanu Sinha \\ Email: uday@econdse.org \\ Delhi School of Economics \\ University of Delhi
}

\section{Working Paper No. 145}

Centre for Development Economics

Department of Economics, Delhi School of Economics 


\title{
On R\&D Information Sharing and Merger
}

\author{
Uday Bhanu Sinh \\ Department of Economics \\ Delhi School of Economics \\ University of Delhi
}

Version: May, 2006

\begin{abstract}
The paper deals with the issue of information sharing in a Cournot duopoly by an innovating firm in the face of a merger with its rival. The innovating firm would share information about the cost realization with its rival provided the market size is relatively small or, the R\&D technology is relatively more efficient in a medium market size. However, in a large market, or in a medium market size with less efficient R\&D technology, the innovating firm does not share information with its rival. We also show that the social welfare may be higher under incomplete information regime.
\end{abstract}

Keywords: Information sharing, market size, R\&D, merger and welfare.

JEL Classifications: L13, O32.

\footnotetext{
$\dagger$ Address for correspondence: Uday Bhanu Sinha, Department of Economics, Delhi School of Economics, University of Delhi, Delhi - 110 007, INDIA.

Email: sinhauday@yahoo.com

${ }^{*}$ I am grateful to Krishnendu Ghosh Dastidar for his help in this paper.
} 


\section{Introduction}

Sharing of private information in oligopoly is an interesting area of research in industrial organization theory (Gal-Or (1987, 1986); Li (1985); Novshek and Sonnenshein (1982); Shapiro (1986); Vives (1990) etc.). In particular, Gal-Or (1986), Li (1985) and Shapiro (1986) concentrated on the Cournot competition characterized by private information about the cost parameters and they find that there exists expected gain associated with the regime of information sharing as compared to a situation of incomplete information. Stenbacka (1991) added an interesting dimension to this information sharing literature by analyzing the issue of R\&D when the innovating firm anticipates a merger. In stenbacka's (1991) paper R\&D outcome is private information of the innovating firm and it was established that for the innovating firm no information sharing is a better strategy. Wong and Tse (1997) clarified the Stenbacka's result and argue that though the innovating firm is better off by not sharing its private information but the investment in cost reduction is not necessarily higher in no information sharing regime and it may be sensitive to the level of technological spillover from the innovating firm to its rival.

We consider a different version of the game on information sharing prior to merger and show that the above result on information sharing is sensitive to the bargaining power at the merger stage. An innovating firm's decision to share information about the cost realization with its rival depends on the market size and the efficiency of R\&D technology. The innovating firm ex-ante commits to share information about the outcome of its R\&D with its rival provided the market size is relatively small and it does not share information at all if the market size is very large. However, for medium market size, the innovating firm likes to share innovation when the R\&D technology is relatively efficient and it does not share information when the R\&D technology is less efficient. Always there is underinvestment in R\&D under both complete and incomplete information regimes as compared to the social optimum. The society is better off due to information sharing than without it when the market size is small or when the $R \& D$ technology is more efficient in a medium market size. Interestingly, the social 
welfare would be higher under incomplete information regime when the market is very large or when the R\&D technology is very inefficient in a medium market size.

The rest of the paper is organized according to the following scheme. Section 2 describes the basic framework of our analysis. Section 3 discusses the choice of information sharing regime and the associated R\&D investment. Welfare comparison is done in section 4. Section 5 concludes the paper.

\section{The Basic Framework}

We closely follow the set up of the model developed by Stenbacka (1991). Consider the following linear inverse demand function: $P=a-Q$, where $P$ is the price of the good, $a$ is a positive constant and $Q$ is the industry output. There are two firms: call them firm 1 and firm 2. Both firms are risk neutral and to begin with they have a common constant marginal cost $\bar{c}$. Firm 1 has monopoly access to an $R \& D$ project, if undertaken this might reduce its marginal cost from $\bar{c}$ to zero with probability $\mathrm{p} \in[0,1]$. Thus, firm 1 fails to innovate the technology with probability $(1-p)$. The cost of $\mathrm{R} \& \mathrm{D}$ for the success probability $p$ is $K p^{2}$, where $\mathrm{K}$ is a positive constant. The parameter $\mathrm{K}$ represents the efficiency of R\&D technology as higher $\mathrm{K}$ represents that for a given probability of success the innovator has to invest more on R\&D. In order to have an interior probability value, we need to impose restriction on the value of $\mathrm{K}$, which we would do later. When firm 1 undertakes R\&D investment, it anticipates the merger possibility after the realization of the R\&D outcome. The exact realization of the R\&D outcome is private information of firm 1; however, firm 2 can observe the level of R\&D expenditure $K p^{2}$. We assume that $a>2 \bar{c}$ in order to ensure that both firms can operate in the market in case firm 1 is successful in R\&D and the merger does not take place. Unlike Stenbacka (1991), we assume away any technological spillover from firm 1 to firm 2 in case of successful R\&D outcome. Also, we change the game by assuming that the non-innovating firm offers to merge by asking a price for its firm.

Now we explicitly lay out the game.

Stage 1: Firm 1 decides whether to commit to share the information about the R\&D outcome with its rival or not. 
Stage 2: Firm 1 undertakes the R\&D and the outcome is realized. The outcome of the innovation is revealed to its rival firm 2 in case firm 1 has committed in stage 1 to share the information. Otherwise, the R\&D outcome is private information of firm 1.

Stage 3: In this stage firm 2 offers to merge with firm 1. Firm 1 either accepts or rejects the offer. In case of rejection both firms compete a la Cournot. In case the merger offer is accepted firm 1 operates in the market under monopoly with the best available technology and firm 2 receives the price it asks in the merger offer.

We assume that firm 1 accepts the merger offer made by firm 2 when its payoff from acceptance is weakly greater than the payoff from rejection. Thus, the essential difference with the papers by Stenbacka (1991) and Wong and Tse (1997) is that here the non-innovating firm is making a take-it-or-leave-it offer and thus having the full bargaining power at the merger stage. We would show that this change of bargaining structure would provide new insights on the issue of information sharing and $R \& D$ of the innovating firm anticipating a merger in a Cournot duopoly. Sharing information credibly is always a problem in the literature on information sharing. However, it is typically assumed that there exists an "outside agency" (say, trade association) through which the information transmission takes place if firm 1 commits to share the information ex-ante.

\section{Analysis of investment in $R \& D$ and information sharing}

Note that firm 1 can choose between two regimes in the first stage of the game. If firm 1 decides not to share information, then the outcome of the R\&D investment remains private information with firm 1. This situation we refer as incomplete information regime. The equilibrium notion we use to solve this regime is perfect Baysian equilibrium. On the other hand, if firm 1 decides to share information then the realization of marginal cost after R\&D would be revealed to firm 2 in stage 2 and as a result the remaining part of the game would be played under complete information. We have to look for subgame perfect equilibrium for this regime of complete information. 


\subsection{Complete information regime}

When firm 1 commits in the first stage of the game to share information about the R\&D outcome then the third stage of the game is played under complete information. Under complete information after the rejection of the merger offer, the Cournot competition takes place and thus, firm 1 and firm 2 receive the following payoffs depending on the success of R\&D.

In case firm 1 is successful in $R \& D$, then the payoffs from duopoly competition for firm 2 and firm 1 are respectively,

$\Pi_{2}^{\mathrm{C}}=\frac{(a-2 \bar{c})^{2}}{9}, \Pi_{1}^{\mathrm{C}}(0)=\frac{(a+\bar{c})^{2}}{9}$

On the other hand, if firm 1 is not successful in R\&D, then the payoffs from duopoly competition for firm 2 and firm 1 are identical and given by,

$$
\Pi_{2}^{\mathrm{C}}=\Pi^{\mathrm{C}}{ }_{1}(\bar{c})=\frac{(a-\bar{c})^{2}}{9}
$$

Thus, firm 2 has to pay at least the above payoffs to firm 1 in order to make the merger offer acceptable to firm 1. After the merger firm 1 operates under monopoly. Given the structure of the game, firm 2 would ask for a price such that firm 1 is indifferent between acceptance and rejection. Thus, firm 2 would ask for a price $\frac{a^{2}}{4}-\frac{(a+\bar{c})^{2}}{9}$ in case firm 1 is successful in R\&D and firm 2 would ask for a price $\frac{(a-\bar{c})^{2}}{4}-\frac{(a-\bar{c})^{2}}{9}$ in case firm 1 is not successful in its R\&D. Once the offer is accepted it would lead to firm 1 receiving the same payoff as it would get by rejection and subsequent duopoly competition. Under complete information, it is obvious that the merger would take place with the above asking prices and the market would be served under monopoly. Фhus, firm 1's net payoff from accepting the merger offer and operating under monopoly is as given in (1) and (2).

Firm 1 would make R\&D investment to maximize the expected payoff, which is given by

\footnotetext{
${ }^{1}$ This result is consistent with Salant et.al. (1983).
} 
$\mathrm{E}\left[\Pi_{1}^{\mathrm{C}}{ }_{1}=p\left\{\frac{(a+\bar{c})^{2}}{9}\right\}+(1-p)\left\{\frac{(a-\bar{c})^{2}}{9}\right\}-K p^{2}\right.$

The maximization of the above function yields the choice of $\mathrm{p}$ under complete information as

$p^{C}=\frac{2 a \bar{c}}{9 K}$.

In order to ensure that the probability $\mathrm{p}^{\mathrm{C}} \leq 1$, we need to assume that

$$
K \geq \frac{2 a \bar{c}}{9} .
$$

\subsection{Incomplete information regime}

Note that without the information sharing, firm 2 would not know the true cost of firm 1, but firm 2 would have a belief about the R\&D outcome in stage 2 of the game. Since firm 2 can observe the investment in R\&D, so it would have a belief that firm 1 is the low cost type (with zero marginal cost) with probability $p$ and the high cost type (with marginal cost $\bar{c}$ ) with probability $(1-p)$. Now firm 2 faces an adverse selection problem in asking a price for merger. If the asking price is too high then neither type of firm 1 would be willing to pay that. Then given this belief structure what firm 2 and firm 1 can expect to get from the subsequent competition (under Baysian Cournot equilibrium) are the following.

$\Pi_{2}^{\mathrm{I}} *=\frac{1}{9}[a-\bar{c}-p \bar{c}]^{2}$

The high cost type firm 1 receives $\Pi_{1}^{\mathrm{I}}(\bar{c})=\frac{(2 a-2 \bar{c}+p \bar{c})^{2}}{36}$

And the low cost type firm 1 receives $\Pi_{1}^{\mathrm{I}}(0)=\frac{(2 a+\bar{c}+p \bar{c})^{2}}{36}$

Firm 2 can offer two kinds of contract in such a situation. In a separating contract firm 2 can ask for a high price which only low cost type firm 1 accepts but the high cost type firm 1 rejects. Alternatively, in a pooling contract firm 2 asks for a merger price which both types of firm 1 accept and the merger takes place.

\footnotetext{
${ }^{2}$ Routine calculation of the Bayesian equilibrium under Counot duopoly will yield these payoffs to firm 2 and firm 1 .
} 
Proposition 1. Pooling contract: In the third stage of the game for $p \leq p^{*}=\frac{(a-\bar{c})}{(a+5 \bar{c})}$, firm 1 asks for a price $\frac{5(a-\bar{c})^{2}}{36}$ and both types of firm 1 accepts the merger offer.

Separating contract: for $p>p^{*}$, firm 2 asks for a price $\frac{(5 a+\bar{c})(a-\bar{c})}{36}$ and only the low cost type firm 1 accepts the offer and the high cost type firm 1 rejects it leading to Cournot duopoly competition between the high cost firm 1 and firm 2.

Proof: First note that if the merger does not take place then the firms will compete as Cournot duopolists and the payoffs are given by (6), (7) and (8).

Define $\bar{R}=\frac{(a-\bar{c})^{2}}{4}-\frac{(a-\bar{c})^{2}}{9}=\frac{5(a-\bar{c})^{2}}{36}$.

Suppose, firm 2 asks for a price $\bar{R}$ for the merger and holds off-the-equilibrium belief in case of rejection that $\mathrm{p}=0$. This offer would be accepted by the high cost type firm 1 as it receives the same payoff by acceptance or rejection. Now to check that this offer will also be accepted by the low cost type firm 1, observe that low cost type firm 1 after rejection has to compete with firm 2 such that firm 2 would produce $\frac{(a-\bar{c})}{3}$ given its posterior off-the-equilibrium belief $\mathrm{p}=0$. Therefore, the optimal output for the low cost type firm 1 to produce in response to that is $\frac{(2 a+\bar{c})}{6}$ leading to a payoff $\frac{(2 a+\bar{c})^{2}}{36}$. In comparison to this, the low cost type firm 1 does better by accepting the offer of merger at price $\bar{R}$ and then running the firm under monopoly to receive $\frac{a^{2}}{4}-\frac{5(a-\bar{c})^{2}}{36}$. Thus, the low cost type firm 1 would also accept this merger offer. This defines pooling contract of the game at the merger stage. In this pooling contract the payoffs to the firms are given below.

Firm 2 receives $\Pi_{2}^{\mathrm{p}}=\bar{R}=\frac{5(a-\bar{c})^{2}}{36}$

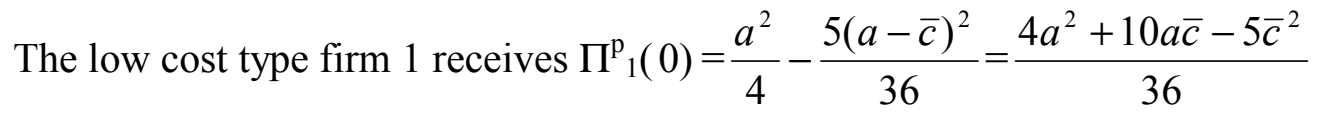


And the high cost type firm 1 receives $\Pi^{\mathrm{p}}{ }_{1}(\bar{c})=\frac{(a-\bar{c})^{2}}{9}$.

Now consider a separating contract such that only the low cost type firm 1 accepts the offer and the high cost type firm 1 rejects it and the firm 2 holds the belief that $p=0$ in case of rejection. Note that the low cost type firm 1 by rejection can get $\frac{(2 a+\bar{c})^{2}}{36}$ by producing optimally in response to firm 2's output with the belief $p=0$. Thus, the asking price $R$ has to be such that low cost type firm 1 receives the above payoff. Hence,

$\frac{a^{2}}{4}-R \geq \frac{(2 a+\bar{c})^{2}}{36}$.

So the firm 2 would ask the optimal price for merger

$\mathrm{R}^{*}=\frac{a^{2}}{4}-\frac{(2 a+\bar{c})^{2}}{36}=\frac{(5 a+\bar{c})(a-\bar{c})}{36}$.

Since the asking price is high (as $R^{*}>\bar{R}$ ), this offer would not be accepted by the high cost type firm 1. Hence, firm 2 has to compete with the high cost type firm 1 and thereby it gets $\frac{(a-\bar{c})^{2}}{9}$. Thus, from the separating contract firm 2 gets the payoff

$\mathrm{p} \frac{(5 a+\bar{c})(a-\bar{c})}{36}+(1-\mathrm{p}) \frac{(a-\bar{c})^{2}}{9}$.

Now by comparing the firm 2's payoff from two kinds of contracts (given by (10) and (14)), we find that firm 2 would offer pooling contract as opposed to a separating contract iff,

$$
\begin{aligned}
& \frac{5(a-\bar{c})^{2}}{36} \geq \mathrm{p} \frac{(5 a+\bar{c})(a-\bar{c})}{36}+(1-\mathrm{p}) \frac{(a-\bar{c})^{2}}{9} \\
& \Rightarrow \mathrm{p} \leq \frac{(a-\bar{c})}{(a+5 \bar{c})}=\mathrm{p}^{*} .
\end{aligned}
$$

Otherwise, for $\mathrm{p}>\mathrm{p} *$, firm 2 offers a separating contract.

The payoff for firm 1 under separating contract is given below.

The low cost type firm 1 receives $\Pi_{1}^{\mathrm{s}}(0)=\frac{(2 a+\bar{c})^{2}}{36}$

And the high cost type firm 1 receives $\Pi_{1}^{\mathrm{s}}(\bar{c})=\frac{(a-\bar{c})^{2}}{9}$. 
Q.E.D.

Note that the high cost type firm 1 receives the same payoff $\frac{(a-\bar{c})^{2}}{9}$ under pooling, separating and complete information regime. However, the low cost type firm 1 receives higher payoff under complete information than the payoff under separating contract (see (1) and (16)). Given these outcomes under complete and incomplete information regimes firm 1 would decide on the R\&D investment and ex-ante sharing of information about the outcome of R\&D. Thus, to determine the choice of information sharing regime and the R\&D investment we need to compare the payoffs from the pooling contract and the complete information regime.

To choose the probability of success under pooling contract, the firm 1 would maximize the following expected payoff.

$\mathrm{E}\left[\Pi_{1}^{\mathrm{I}}\right]=\mathrm{p} \Pi^{\mathrm{p}}{ }_{1}(0)+(1-\mathrm{p}) \Pi^{\mathrm{p}}{ }_{1}(\bar{c})-K p^{2}$

Now, with the firm 1's payoffs defined under pooling contract (given by (11) and (12)), we find that the maximisation of the expected payoff from $R \& D$ yields the optimal choice of $\mathrm{R} \& \mathrm{D}$ success probability as

$\mathrm{p}^{\mathrm{p}}=\frac{\bar{c}(2 a-\bar{c})}{8 K}$.

First, by comparing $\Pi^{\mathrm{C}}{ }_{1}(0)$ and $\Pi^{\mathrm{p}}{ }_{1}(0)$ (from (1) and (11)) we find that $\Pi^{\mathrm{C}}{ }_{1}(0)>\Pi^{\mathrm{p}}{ }_{1}(0)$ when $\frac{9}{2} \bar{c}>a>2 \bar{c}$. So, $\mathrm{p}^{\mathrm{C}}>\mathrm{p}^{\mathrm{p}}$ and hence, the expected payoff to firm 1 is greater under complete information than under pooling contract (from (3) and (18)). So, firm 1 would choose to share information and the probability of successful R\&D is given by (4).

Second, for $a>\frac{9}{2} \bar{c}, \Pi_{1}^{\mathrm{C}}(0)<\Pi^{\mathrm{p}}{ }_{1}(0)$. However, to have the pooling equilibrium in the third stage of the game we need the choice of $\mathrm{p}^{\mathrm{p}} \leq \mathrm{p}^{*}$. This leads to the condition that $\mathrm{K}>\frac{\bar{c}(2 a-\bar{c})(a+5 \bar{c})}{8(a-\bar{c})}=\bar{k}$ (say).

Observe that for $\mathrm{K}>\bar{k}, \mathrm{p}^{\mathrm{p}}>\mathrm{p}^{\mathrm{C}}$ and hence the expected payoff under pooling contract would be higher than the payoff from complete information. Now for $\mathrm{K}<\bar{k}$, firm 1 can either choose 
not to share information and stick to the choice $\mathrm{p}^{*}$ at the R\&D stage to enforce the pooling contract in the merger stage or it can choose to share information and choose R\&D investment optimally under complete information given by (3). Note that

$\mathrm{p}^{\mathrm{c}}=\mathrm{p}^{*}$ for $\mathrm{K}=\frac{2 a \bar{c}(a+5 \bar{c})}{9(a-\bar{c})}$

Thus, for $\frac{2 a \bar{c}(a+5 \bar{c})}{9(a-\bar{c})}<\mathrm{K}<\bar{k}$, the choice of $\mathrm{p}^{\mathrm{c}}<\mathrm{p}^{*}$.

Now firm 1 would receive by choosing $\mathrm{p}^{*}$ and subsequently enforcing the pooling contract $\mathrm{p}^{*}\left\{\frac{4 a^{2}+10 a \bar{c}-5 \bar{c}^{2}}{36}\right\}+\left(1-\mathrm{p}^{*}\right)\left\{\frac{(a-\bar{c})^{2}}{9}\right\}-K p^{* 2}$.

And firm 1's payoff under complete information for $\frac{2 a \bar{c}(a+5 \bar{c})}{9(a-\bar{c})} \leq \mathrm{K}<\bar{k}$ would be less than the payoff from pooling contract as $\mathrm{p}^{\mathrm{c}} \leq \mathrm{p}^{*}$ and $\Pi^{\mathrm{C}}{ }_{1}(0)<\Pi^{\mathrm{p}}{ }_{1}(0)$.

Consider the lowest permissible value of $K=\frac{2 a \bar{c}}{9}$, where under complete information regime the firm 1 would choose $p^{c}=1$. From (3), the firm 1 would receive the payoff $=\frac{(a+\bar{c})^{2}}{9}-\frac{2 a \bar{c}}{9}$.

On the other hand, at $K=\frac{2 a \bar{c}}{9}$ by sticking to the choice of $\mathrm{p}^{*}$ under incomplete information the firm 1 would receive $\mathrm{p}^{*}\left\{\frac{4 a^{2}+10 a \bar{c}-5 \bar{c}^{2}}{36}\right\}+\left(1-\mathrm{p}^{*}\right)\left\{\frac{(a-\bar{c})^{2}}{9}\right\}-\frac{2 a \bar{c}}{9} p^{* 2}$. Plugging the value of $\mathrm{p}^{*}$ from (15), one can easily compare the payoffs between complete information and pooling contract. Now there are two possibilities. If $a<13.1085 c^{3}$, the payoff under complete information is strictly greater than the payoff from pooling contract. Thus, by continuity of payoffs there exists a $\mathrm{K}=k$ such that the payoff from complete information is exactly equal to the payoff from pooling contract at $\mathrm{p}^{*}$ and $\frac{2 a \bar{c}}{9}<k<\frac{2 a \bar{c}(a+5 \bar{c})}{9(a-\bar{c})}$. So, for

${ }^{3}$ At $K=\frac{2 a \bar{c}}{9}$ the firm 1 receives higher payoff under complete information as compared to the payoff from the pooling contract iff $2 a^{3}+45 \bar{c}^{3}<a^{2} \bar{c}+334 a \bar{c}^{2}$, which means $a<13.1085 \bar{c}$ approximately. 
$\mathrm{K} \geq k$, firm 1 would not share information and pooling equilibrium would be the outcome. On the other hand for $\frac{2 a \bar{c}}{9}<\mathrm{K}<k$, firm 1 would like to share the information. The last possibility is when $a \geq 13.1085 \bar{c}$. Here the payoff from pooling contract always dominates the payoff from the complete information. Hence, firm 1 would not like to share information for very large market.

To sum up,

Proposition 2: (a) Suppose $\frac{9}{2} \bar{c} \geq a>2 \bar{c}$, firm 1 receives higher expected payoff under complete information than incomplete information. Hence, firm 1 would commit to share information about its $R \& D$ outcome to its rival.

(b) Suppose $13.1085 \bar{c}>a>\frac{9}{2} \bar{c}$, then for $\frac{2 a \bar{c}}{9} \leq K<\hat{k}$, firm 1 receives higher payoff under complete information than incomplete information. And for $K \geq \hat{k}$, firm 1 receives higher payoff under incomplete information than complete information. Hence, for $K \geq k$, firm 1 would choose not to share information with its rival, and for $\frac{2 a \bar{c}}{9} \leq K<\hat{k}$ firm 1 would choose to share information with its rival.

(c) For $a \geq 13.1085 \bar{c}$, firm 1 receives higher payoff from pooling contract than form complete information. Hence, firm 1 would never like to share information about its R\&D outcome to its rival.

Observe that merger takes place both under pooling equilibrium and complete information regime. Now, given the firm 1's choice of information sharing regime described above, the following proposition characterizes the choice of R\&D success probability for firm 1.

Proposition 3: (a) For $\frac{9}{2} \bar{c} \geq a>2 \bar{c}$, firm 1 chooses $p^{C}=\frac{2 a \bar{c}}{9 K}$

(b) For $13.1085 \bar{c}>a>\frac{9}{2} \bar{c}$, firm 1 chooses $p^{p}=\frac{\bar{c}(2 a-\bar{c})}{8 K}$ when $K>\bar{k}$;

firm 1 chooses $p^{p}=p^{*}$ when $\bar{k} \geq K \geq k$, and firm 1 chooses $p^{C}=\frac{2 a \bar{c}}{9 K}$ when $k>\mathrm{K} \geq \frac{2 a \bar{c}}{9}$. 
(c) For $a \geq 13.1085 \bar{c}$, firm 1 chooses $p^{p}=\frac{\bar{c}(2 a-\bar{c})}{8 K}$ when $K \geq \bar{k}$ and firm 1 chooses $p^{p}=p^{*}$ when $\frac{2 a \bar{c}}{9} \leq K<\bar{k}$.

The above findings on $\mathrm{R} \& \mathrm{D}$ choice and information sharing for $13.1085 \bar{c}>a>\frac{9}{2} \bar{c}$ can be represented in the following diagram.

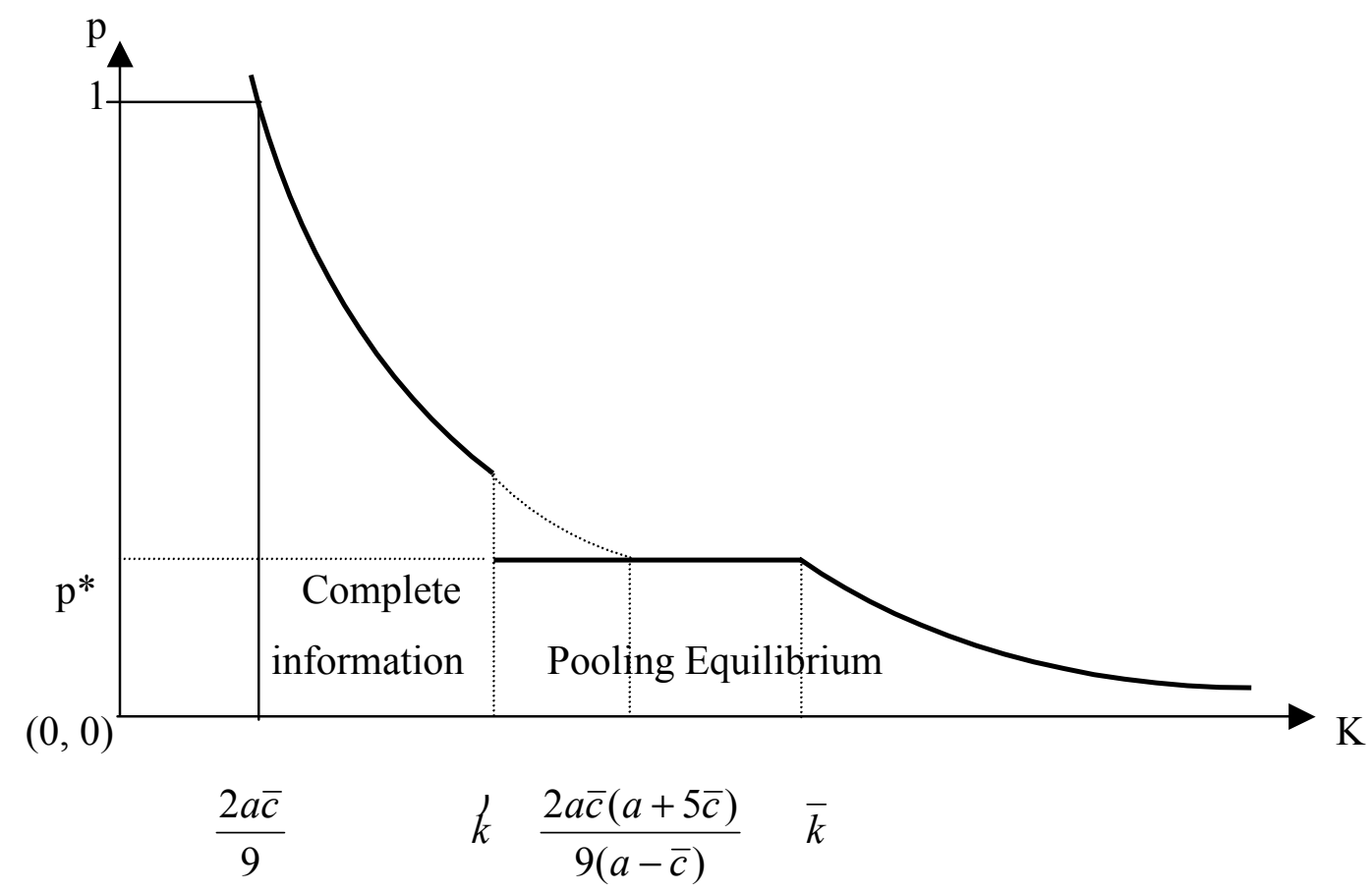

Figure 1. Choice of information regime and $R \& D$ success probability for medium market size $\left(13.1085 \bar{c}>a>\frac{9}{2} \bar{c}\right)$.

It should be noted here that for relatively small market size $\left(\frac{9}{2} \bar{c}>a>2 \bar{c}\right)$, firm 1 always likes to share information about its R\&D outcome ex-ante and the merger takes place under the complete information. The probability of successful R\&D is also higher for this range of parameters. However, for medium market size (i.e., $13.1085 \bar{c}>a>\frac{9}{2} \bar{c}$ ) the behaviour of firm 1 regarding information sharing and $R \& D$ investment is rather more interesting. The innovating firm has incentive to share information prior to merger when the R\&D technology 
is more efficient and firm 1 does not like to share information when the R\&D technology is less efficient. When the market size is very large $(a \geq 13.1085 \bar{c})$ then firm 1 never likes to share information about R\&D outcome with its rival. Thus, within the framework of single model we are able to show both information sharing and not sharing as possible outcomes. This finding is contrary to Stenbacka (1991) and Wong and Tse (1997), where the innovating firm, having the full bargaining power, never likes to share information with its rival. The difference in the results mainly stem form the fact that the merger offer made by firm 2 (noninnovating firm) gives it the full bargaining power over the merger gain given the information constraint. This result is also different from the other standard models on information sharing under Cournot competition with private information about costs, where the typical result is to share information (Gal-Or (1986), Li (1985) and Shapiro (1986).

The intuition behind the choice of information regime is straightforward. The decision of information sharing crucially depends on the size of the market as the information rent received by the low cost type firm 1 under pooling contract increases with the increase in market size. For smaller market size, the information sharing is a dominant strategy for firm 1. However, for medium market size the choice of information sharing depends on the efficiency of R\&D technology. First, note that for medium size of the market the pooling equilibrium leads to a moderate amount of information rent to the low cost type firm 1. Note that for more efficient $R \& D$ technology the likelihood of receiving the better technology is high for firm 1 given any level of R\&D investment. In such a situation with the likelihood of receiving better technology with high probability it is optimal for firm 1 to share information and enforce the complete information outcome in the merger stage. In this case under incomplete information either firm 1 has to choose low probability of success to enforce pooling contract or a high probability of success leading to a separating contract. Choosing low probability of success yields lower payoff to firm 1 given the moderate information rent. Moreover, in separating equilibrium in the merger stage, firm 2's off-the-equilibrium belief of $\mathrm{p}=0$ forces firm 1 to accept merger offer at an asking price which is higher than the complete information regime leading to a lower payoff to the low cost type firm 1. When the market size is very large the information rent under pooling contract is so large that firm 1 does not 
like to share information even though it sticks to the choice of $\mathrm{p}^{*}$ for efficient R\&D technology.

Interestingly, for medium and large market, there exists a range of choice of a constant $\mathrm{p}^{*}$ despite the fact that the efficiency of the R\&D technology varies over a range. This phenomenon leads to underinvestment in $\mathrm{R} \& \mathrm{D}$ by firm 1 to enforce the pooling equilibrium in the incomplete information regime. In particular, in a medium market size, for $k<\mathrm{K}<\frac{2 a \bar{c}(a+5 \bar{c})}{9(a-\bar{c})}$ the $\mathrm{R} \& \mathrm{D}$ investment under incomplete information regime is even lower than the complete information regime. Note that the consumer welfare only depends on the probability of successful R\&D. Consumers are always served under monopoly due to merger. If the probability of success is higher then the consumers are served with better technology with higher probability leading to higher expected consumers surplus. Therefore, the underinvestment in $\mathrm{R} \& \mathrm{D}$ under incomplete information is bad for the consumers as compared to complete information when $k \leq \mathrm{K}<\frac{2 a \bar{c}(a+5 \bar{c})}{9(a-\bar{c})}$ in a medium market size or when $\frac{2 a \bar{c}}{9} \leq$ $K<\frac{2 a \bar{c}(a+5 \bar{c})}{9(a-\bar{c})}$ in a large market. On the other hand, when the R\&D technology is more inefficient i.e., $\mathrm{K} \geq \frac{2 a \bar{c}(a+5 \bar{c})}{9(a-\bar{c})}$, the consumers are better off under incomplete information regime both in medium and large market.

\section{Welfare Analysis}

We now look at the social welfare, which is the sum of consumers and producers surplus. The social welfare in case of successful R\&D can be calculated to be $\frac{3 a^{2}}{8}$ and the social welfare in case of unsuccessful $\mathrm{R} \& \mathrm{D}$ is $\frac{3(a-\bar{c})^{2}}{8}$. Thus, the expected social welfare is given as $p\left\{\frac{3 a^{2}}{8}\right\}+(1-p)\left\{\frac{3(a-\bar{c})^{2}}{8}\right\}-K p^{2}$ 
This expression is maximized at $p^{w}=\frac{3\left(2 a \bar{c}-\bar{c}^{2}\right)}{16 K}$.

By comparing $\mathrm{p}^{\mathrm{C}}$ and $\mathrm{p}^{\mathrm{w}}$, we find that $\mathrm{p}^{\mathrm{C}}<\mathrm{p}^{\mathrm{w}}$ given our assumption $a>2 \bar{c}$. Also, $\mathrm{p}^{\mathrm{p}}<\mathrm{p}^{\mathrm{w}}$ always holds. Thus, both under complete and incomplete information regimes the investment in R\&D is always less than the socially optimum level of investment. Given the concavity of the social welfare function, the comparison of welfare between complete and incomplete information regimes depends on which regime generates higher probability of success in R\&D.

First note that for relatively small market $\left(\frac{9}{2} \bar{c}>a>2 \bar{c}\right)$, the welfare is always higher under complete information regime. However, when the market size is medium (i.e., $13.1085 \bar{c}>a>\frac{9}{2} \bar{c}$ ), for $\frac{2 a \bar{c}}{9} \leq K<\hat{k}$ the welfare is higher under complete information. For $\frac{2 a \bar{c}(a+5 \bar{c})}{(a-\bar{c})}>K \geq \hat{k}$, since the investment in $\mathrm{R} \& \mathrm{D}$ is less under incomplete information, hence the welfare is lower under the incomplete information regime as compared to the complete information regime. However, for $\mathrm{K} \geq \frac{2 a \bar{c}(a+5 \bar{c})}{(a-\bar{c})}$, the $\mathrm{R} \& \mathrm{D}$ success probability is higher under incomplete information regime and hence, the welfare is higher under incomplete information regime as compared to the complete information regime. For very large market under incomplete information the welfare is higher for $\mathrm{K} \geq \frac{2 a \bar{c}(a+5 \bar{c})}{(a-\bar{c})}$, but the welfare is lower for $\frac{2 a \bar{c}}{9} \leq K<\frac{2 a \bar{c}(a+5 \bar{c})}{(a-\bar{c})}$.

To sum up the above discussion,

Proposition 4. (a) For small market, the welfare is higher under complete information regime.

(b) For medium market size when the $R \& D$ technology is more efficient (i.e., $K<k$ ), the social welfare is higher under complete information regime than under incomplete information. For intermediate efficiency of $R \& D$ technology (i.e., for $\frac{2 a \bar{c}(a+5 \bar{c})}{(a-\bar{c})}>K \geq k$ ) the 
social welfare is lower under incomplete information regime. However, for very inefficient $R \& D$ technology (i.e., $\left.K \geq \frac{2 a \bar{c}(a+5 \bar{c})}{(a-\bar{c})}\right)$, the social welfare would be higher under incomplete information regime than under complete information.

(c) In a large market under incomplete information the social welfare would be higher for $K \geq$ $\frac{2 a \bar{c}(a+5 \bar{c})}{(a-\bar{c})}$, but it would be lower for $\frac{2 a \bar{c}}{9} \leq K<\frac{2 a \bar{c}(a+5 \bar{c})}{(a-\bar{c})}$.

\section{Conclusion}

In this paper we have dealt with the issue of information sharing in the context of Cournot duopoly in the face of a merger. An innovating firm might ex-ante commit to share its private information about the cost realization when it anticipates a merger possibility prior to the market competition stage. It is found that for relatively small market the innovating firm would always like to share information about its R\&D outcome ex-ante and the incentive for innovation would also be higher under complete information. For medium market size, the decision to share information depends on the efficiency in $R \& D$ technology. When the R\&D technology is relatively more efficient, the innovating firm shares information and the incentive for $R \& D$ is higher under the information sharing regime. On the other hand, when the R\&D technology is relatively less efficient, the firm decides not to share information. Though for an intermediate level of R\&D efficiency the incomplete information regime leads to less R\&D investment than the complete information regime, but for high degree of inefficiency of R\&D technology the incomplete information regime provides higher incentive for $R \& D$ investment. For very large market, innovating firm would never like to share information about its R\&D outcome with its rival. However, the incentive for innovation is lower under incomplete information regime for more efficient $R \& D$ technology. Thus, innovating firm's decision to share information about the cost realization with its rival prior to merger depends on its bargaining power, market size and the efficiency of R\&D technology.

Comparing with the socially desirable level of $R \& D$ investment, we find that the $R \& D$ investment is always less than the social optimum both under complete and incomplete information regimes. For small market, the expected social welfare is higher under 
information sharing than without it. For medium market size, it is found that when the R\&D technology is relatively more efficient then the expected social welfare is higher under information sharing regime and when the R\&D technology is very inefficient then the expected social welfare would be higher under the incomplete information regime. However, for the intermediate level of efficiency of R\&D technology, though the decision of the innovating firm is not to share information but the R\&D investment is less, leading to lower welfare under incomplete information regime as compared to the information sharing regime. For large market under incomplete information regime the social welfare would be higher when the R\&D technology is less efficient, but the social welfare would be lower for more efficient R\&D technology as compared to the complete information regime. 


\section{References}

Gal-or, E., 1986. Information transmission - Cournot and Bertrand equilibria. Review of Economic Studies 53, 85-92.

Gal-or, E., 1987. First mover disadvantages with private information. Review of Economic Studies 54, 279-292.

Li, L., 1985. Cournot oligopoly with information sharing. Rand Journal of Economics 16, 521-536.

Novshek, W., Sonnenschein, H., 1982. Fulfilled expectations, Cournot duopoly with information acquisition and release. Bell Journal of Economics 13, 214-218.

Salant, S. W., Switzer, S., Reynolds, R. J., 1983. The losses of horizontal merger: The effects of an exogenous change in industry structure on Cournot - Nash equilibrium. Quarterly Journal of Economics 98,185 - 99.

Shapiro, C., 1986. Exchange of cost information in Oligopoly. Review of Economic Studies $53,433-446$.

Stenbacka, L. R., 1991. Mergers and investments in cost reduction with private information. International journal of Industrial organization 9, 397-405.

Wong, K. P., Tse, M. K. S., 1997. Mergers and investments in cost reduction with private information revisited. International journal of Industrial organization 15, 629-634.

Vives, X., 1990. Information and competitive advantage. International Journal of Industrial Organization 8, 17-35. 